\title{
Determining the Efficiency of Different Preoperative Difficult Intubation Tests on Patients Undergoing Caesarean Section
}

\author{
İlker Yıldırım1, Mehmet Turan İnal2, Dilek Memiş2, F. Nesrin Turan³ \\ ${ }^{1}$ Clinic of Anesthesiology, Uzunköprü Public Hospital, Edirne, Turkey \\ ${ }^{2}$ Department of Anesthesiology and Reanimation, Trakya University School of Medicine, Edirne, Turkey \\ ${ }^{3}$ Department of Biostatistics, Trakya University School of Medicine, Edirne, Turkey
}

Background: Pregnancy-induced anatomical and physiological changes in the airway make airway management difficult in obstetric patients; thus, preoperative evaluation of the airway is important for obstetric patients.

Aims: To determine the effectiveness of the modified Mallampati test; the interincisor, sternomental and thyromental distances and the upper limb bite test. The second aim was to assess the effectiveness of the combination of the upper limb bite test with the other tests in obstetric patients.

Study Design: Cross-sectional study.

Methods: Pregnant women $(n=250)$ scheduled for caesarean section were analysed. The patients' ages, heights and weights were collected. Preoperative airway evaluation was done by using a modified version of the Mallampati test. The interincisor, sternomental and thyromental distances were measured, and the upper limb bite test was performed. The laryngoscopy difficulty was evaluated by using Cormack-Lehane classification.

Results: No statistically significant differences were found between groups in age, height or weight $(\mathrm{p}>0.05)$. The modified Mallampati test and interincisor, sternomental and thyromental distances revealed a lower number of easy intubations than that determined by the Cormack-Lehane classification and a higher number of difficult intubations than the actual number of cases $(\mathrm{p}<0.05)$. The sensitivity and specificity of the modified Mallampati test, the upper limb bite test, the interincisor distance test and the sternomental and thyromental distance tests were found to be $73.08,57.69,84.62,80.77$ and 88.46 and 90.62, 99.11, 83.04, 84.37 and 87.05, respectively. When the combinations were examined, the sensitivity and specificity of the combination of the upper limb bite test with the modified Mallampati test were found to be 57.69 and 100 , respectively. When the upper limb bite test was combined with the interincisor distance, the sensitivity and specificity were 46.15 and 100 , respectively. We found a sensitivity and specificity of 93.75 and 95.30 , respectively, for the combination of the upper limb bite test with the thyromental distance test. The sensitivity and specificity of the combination of the upper limb bite test with the modified Mallampati test and interincisor distance test were found to be 46.15 and 100 , respectively. For combination of all the tests, the sensitivity and specificity was 42.31 and 100 , respectively.

Conclusion: When all combinations are evaluated in the decision of difficult intubation, the combination of the upper limb bite test and thyromental distance test is superior to the use of other methods alone to predict difficult intubation in pregnant women.

Keywords: Caesarean section, difficult intubation, predictive tests

\footnotetext{
Address for Correspondence: Dr. Mehmet Turan İnal, Department of Anesthesiology and Reanimation, Trakya University School of Medicine, Edirne, Turkey Phone: +90 $2842357641 \quad$ e-mail: mehmetturaninal@yahoo.com Received: 19 September 2016 Accepted: 2 April 2017 • DOI: 10.4274/balkanmedj.2016.0877

Available at www.balkanmedicaljournal.org

Cite this article as:

Yıldırım İ, İnal MT, Memiş D, Turan FN. Determining the Efficiency of Different Preoperative Difficult Intubation Tests on Patients Undergoing Caesarean Section. Balkan Med J 2017;34:436-43

${ }^{\circ}$ Copyright 2017 by Trakya University Faculty of Medicine / The Balkan Medical Journal published by Galenos Publishing House.
} 
Difficult intubation is seen more often in obstetric patients than in other surgical patients and is the leading cause of anaesthesia-related maternal mortality. Pregnancy-induced anatomical and physiological changes in the airway make airway management in obstetric patients one of the important issues in anaesthesia practice. This condition arises from limited laryngoscopy movement due to oedema in the airways, a large tongue, fragile tissues and an enlarged abdomen. Failed intubation is very important in this group of patients in terms of increased morbidity and mortality. In the literature, considering pregnancy-related deaths, complications associated with anaesthesia practice are responsible for $2.5 \%$ of all maternal deaths, of which airway problems constitute $58 \%$, of which endotracheal intubation failures constitute a major part (1-3). Therefore, the preoperative evaluation of the difficulty of intubation is important for patients undergoing caesarean section under general anaesthesia (4-6).

A variety of different tests are used to evaluate difficult intubation in advance. Among them are the Upper Lip Bite test (ULBT), the Modified Mallampati test (MMT), thyromental distance (TMD) measurement, sternomental distance (SMD) measurement and interincisor distance (IID) measurement. Several previous studies reported on the effectiveness and weaknesses of these tests (7-12). Different studies (8-13) concluded that the ULBT test is simple and easy to apply; therefore, we planned to combine the other tests with the ULBT test.

The first aim of the study was to determine the effectiveness of each of the single difficult intubation predictive tests, and the second aim was to determine the effectiveness of the combination of different predictive tests with the ULBT test in patients undergoing caesarean section under general anaesthesia.

\section{MATERIALS AND METHODS}

The study was carried out with a total of two hundred-fifty pregnant women planned for caesarean section. Written informed consent was obtained between the dates 10.06.2011 and 01.09.2012. Ethical approval for this study (Trakya University ethical committe number: 2011/113) was provided on 08.06.2011.

Patients who had undergone neck or jaw surgery, burn or trauma related to the upper respiratory tract; had a condition leading to limited cervical mobility such as cervical disc herniation or rheumatoid arthritis; had ankylosing spondylitis or had a bulk in the pharynx, larynx or mouth were not included in the study.

The MMT, IID, SMD, TMD and ULBT tests were performed on the patients in the preoperative period and recorded by a research assistant (IY) who had at least 2 years of experience in anaesthesia. In addition, data on age, height and weight of the patients were collected.

The MMT as described by Samsoon and Young (7) classified the airway into four classes.

Class I: Soft palate, fauces, uvula and pillars visible, Class II: Soft palate, fauces and base of uvula visible, soft palate visible and hard palate visible. Classes I and II were predictive of easy intubation, and Classes III and IV were predictive of difficult intubation.

The IID was defined as the distance between the incisors when the patient's mouth was completely open, and less than 3-3.5 $\mathrm{cm}$ was regarded as a sign of a difficult intubation (8-11).

The SMD was defined as the distance between the middle point of the jaw and the upper limit of the manibrium sterni while the patient's head was in full extension, and the mouth was closed. Less than $12.5 \mathrm{~cm}$ was regarded as a sign of a difficult intubation (8-11).

The TMD was defined as the distance between the middle point of the tip of the jaw and the overhang of the thyroid cartilage while the patient's head was in full extension, and the mouth was closed; less than $6 \mathrm{~cm}$ was regarded as a difficult intubation (8-11).

The ULBT was described by Khan et al. (12) in 2003. In this test, Class 1: The lower incisors can bite the upper lip above the vermilion line. Class 2: The lower incisors can bite the upper lip below the vermilion line. Class 3: The lower incisors cannot bite the upper lip. Classes I and II were predictive of easy intubation, and Class III was predictive of a difficult intubation.

After pregnant women received general anaesthesia with 2 $\mathrm{mg} / \mathrm{kg}$ propofol (Propofol; Fresenius Kabi medical, Hamburg, Germany) and $1 \mathrm{mg} / \mathrm{kg}$ succinylcholine (lysthenon; Fako Medical, İstanbul, Turkey) as in a standard in intubation, a rapid intubation was performed. Intubation was performed by one anaesthetist who was blinded to the study, using the Macintosh blade, and laryngoscopy difficulty was evaluated by the Cormack-Lehane classification, without applying cricoid pressure. Grade I: Full view of the glottis can be seen, Grade II: Partial view of the glottis can be seen, Grade III: Only the epiglottis can be seen and Grade IV: The epiglottis and glottis cannot be seen. Grades I and II were accepted as an easy airway, and Grades III and IV were accepted as a difficult airway. Patients were classified as easy intubations or difficult intubations using the Cormack grading system. Accordingly, those who had Cormack Grade I-II airways were classified as easy intubations, and those who had Cormack Grade III-IV airways were classified as difficult intubations (14). 
Difficult intubation, as defined by the American Association of Anaesthetists, is three or more failed attempts during intubation or the process taking more than 10 minutes (15). Between failed intubation attempts, oxygen was given by mask ventilation to the patient. In the case of tracheal ventilation failure, mask ventilation or laryngeal mask airway was used for the patient. Bleeding, lacerations, dental trauma and airway trauma were all recorded.

The first aim was to determine the effectiveness of the single difficult intubation predictive tests and the second aim was to determine the effectiveness of the combination of different predictive tests with the ULBT test due to its simplicity and non-invasiveness. When both tests were positive in determining difficult intubation, the combined test was considered positive.

\section{Statistical analysis}

Statistical evaluation was carried out using SPSS Statistics for Windows, Version 19.0. (IBM Corp., Armonk, NY: Released 2010). After the compliance of the data with the normal distribution was checked by a one-sample KolmogorovSmirnov test, a Mann-Whitney U test was used for nonnormally distributed data. For the interincisor distance, sternomental distance and thyromental distance measurements, cut-off points were calculated by receiver operating curve analysis. The Kappa test for compliance between CormackLehane classification and MMT, ULBT, IID, SMD and TMD was used, and the McNemar test was used for the difference between tests. Mean \pm standard deviation were used, and results were considered significant at $\mathrm{p}<0.05$. Post hoc evaluation indicated that a sample size of 248 subjects would achieve $87 \%$ power to detect a difference of -0.577 between two diagnostic tests with sensitivities of 0.423 and 1.000 . This procedure uses a two-sided McNemar test with a significance level of 0.05 . The prevalence of disease in the population is 0.104 . The proportion of discordant pairs is 0.940 .

\section{RESULTS}

Two hundred-fifty female patients were included in the study. Patients were divided into two groups according to the Cormack-Lehane classification. A total of 224 (89.6\%) patients were classified as easy intubations and $26(10.4 \%)$ were classified as difficult intubations. All patients in the difficult intubation group were successfully ventilated, and in 4 of the 26 patients, blood was detected in the laryngoscope. No lacerations, dental trauma or airway trauma were recorded.

Mean patient age was $30.04 \pm 6.81$ years in the easy intubation group and $30.42 \pm 6.92$ years in the difficult intubation group.
Mean patient height was $162.33 \pm 6.47 \mathrm{~cm}$ in the easy intubation group and $160.38 \pm 8.12 \mathrm{~cm}$ in the difficult intubation group. Mean patient weight was $81.00 \pm 17.24 \mathrm{~kg}$ in the easy intubation group and $82.12 \pm 20.44 \mathrm{~kg}$ in the difficult intubation group. When the groups were compared, no statistically significant difference was found between groups in age, height, or weight (p>0.05) (Table 1).

When the difficult intubation group was assessed by MMT, $203(81.20 \%)$ of 224 patients were identified as easy intubations while $21(8.40 \%)$ were identified as difficult intubations. Seven $(2.80 \%)$ out of 26 patients were assessed as easy intubations and 19 of them (7.60\%) were assessed as difficult intubations. According to IID measurements, $186(74.40 \%)$ out of 224 patients were identified as easy intubations, and $38(1.60 \%)$ were identified as difficult intubations. Four $(1.60 \%)$ out of 26 patients were assessed as easy intubations, and $22(8.80 \%)$ were assessed as difficult intubations. We found the cut-off point for the IID test to be $4.5 \mathrm{~cm}$. The AUC value was 0.926 . When SMD was used, $189(75.60 \%)$ out of 224 patients were identified as easy intubations, and $35(14.00 \%)$ were identified as difficult intubations. Five $(2.0 \%)$ out of 26 patients were assessed as easy intubations, and the other $21(8.4 \%)$ were assessed as difficult intubations. In our study, the cut-off point for the SMD test was $13.5 \mathrm{~cm}$. The AUC value for SMD was 0.888 . According to TMD measurements, 195 (78.0\%) out of 224 patients were identified as easy intubations and 29 (11.6\%) were identified as difficult intubations. Three (1.2\%) out of 26 patients who were classified as difficult intubations according to the Cormack-Lehane classification were identified as easy intubations, and 23 of them $(9.2 \%)$ were assessed as difficult intubations. The cut-off point for the TMD was $6.5 \mathrm{~cm}$. The AUC was 0.901 . When the difficult intubation group was assessed by ULBT, $222(88.80 \%)$ out of 224 patients were identified as easy intubations, and $2(0.80 \%)$ of them were identified as difficult intubations. Eleven $(4.40 \%)$ patients were assessed as easy intubations, and $15(6.00 \%)$ were assessed as difficult intubations. When the groups were compared statistically, the MMT, IID, SMD and TMD revealed a lower number than the case number of easy intubations and a higher number than the case number of difficult intubations as determined by the Cormack-Lehane

TABLE 1. Demographic data

\begin{tabular}{lccc}
\hline & $\begin{array}{c}\text { Easy intubation group } \\
(\mathrm{n}=224)\end{array}$ & $\begin{array}{c}\text { Difficult intubation group } \\
(\mathrm{n}=26)\end{array}$ & $\mathrm{p}$ \\
\hline Age (years) & $30.04 \pm 6.81$ & $30.42 \pm 6.92$ & 0.628 \\
Height $(\mathrm{cm})$ & $162.33 \pm 6.47$ & $160.38 \pm 8.12$ & 0.209 \\
Weight $(\mathrm{kg})$ & $81.00 \pm 17.24$ & $82.12 \pm 20.44$ & 0.400 \\
\hline
\end{tabular}


classification. The ULBT revealed a higher number than the case number of easy intubations and a lower number than the case number of difficult intubations $(\mathrm{p}<0.05)$ (Table 2$)$. The sensitivity, specificity and positive and negative predictive values are shown in Table 3.

The relationships of all intubation tests with difficult intubations by having two or more combinations with the ULBT were investigated statistically. When the ULBT and MMT were combined, 11 cases of easy intubation were identified as difficult intubations, while 15 cases were found to have the probability of difficult intubation with the binary test. The difference between the possible cases of difficult intubation and the actual number of difficult intubations was found to be statistically significant $(p=0.001$, Table 4$)$ The sensitivity, specificity, PPV and NPV were 57.69\%, 100\%, $100 \%$ and $95.32 \%$, respectively (Table 5). If ULBT and IID were combined, in the binary test, 14 cases of easy intubation were identified as difficult intubations, and 12 cases were identified as probable difficult intubations. A statistically significant difference was detected between possible cases of difficult intubation and the actual number of difficult intubations $(\mathrm{p}<0.001$, Table 4$)$. We found the sensitivity, specificity, PPV and NPV to be $46.15 \%, 100 \%, 100 \%$ and $94.12 \%$, respectively (Table 5). When ULBT and SMD were combined, 12 cases of easy intubation were identified as difficult intubations, while 15 cases were identified as probable difficult intubations. The difference between the possible cases of difficult intubation and the actual number of difficult intubations was statistically significant $(\mathrm{p}=0.003$, Table 4). The sensitivity was $53.85 \%$, specificity was $99.55 \%$, PPV was 93.33 and NPV was $94.89 \%$ (Table 5). If ULBT and TMD were combined, 11 cases of easy intubation were identified as difficult intubations, while 16 cases were identified as probable difficult intubations. A statistically significant difference was detected between the possible cases of difficult intubation and the actual number of difficult intubations $(p=0.006$, Table 4). The sensitivity, specificity, PPV and NPV were 93.75\%, 95.30\%, 57.69 and 99.55\%, respectively (Table 5).

In the statistical comparisons using Cormack grade performed with the ULBT, MMT and IID, in the ternary test, 14 cases of easy intubation were identified as difficult intubations, while

TABLE 2. Comparison of the groups according to the MMT, ULBT, IID, SMD and TMD

\begin{tabular}{|c|c|c|c|c|c|c|c|}
\hline & \multicolumn{2}{|c|}{$\begin{array}{c}\text { Easy intubation (Cormack } \\
\text { Grade I-II) }\end{array}$} & \multicolumn{2}{|c|}{$\begin{array}{c}\text { Difficult intubation (Cormack } \\
\text { Grade III-IV) }\end{array}$} & \multicolumn{2}{|c|}{ Total } & \multirow[t]{2}{*}{$\mathrm{p}^{*}$} \\
\hline & $\mathrm{n}$ & $\%$ & $\mathrm{n}$ & $\%$ & $\mathrm{n}$ & $\%$ & \\
\hline Difficult intubation (MMT III-IV) & 21 & 8.40 & 19 & 7.60 & 40 & 16.00 & $0.014 *$ \\
\hline Easy intubation (ULBT I-II) & 222 & 88.80 & 11 & 4.40 & 233 & 93.20 & $0.022 *$ \\
\hline Difficult intubation (IID $\leq 4.5 \mathrm{~cm})$ & 38 & 15.2 & 22 & 8.8 & 60 & 8.8 & $<0.001 *$ \\
\hline Easy intubation $(\mathrm{SMD}>13.5 \mathrm{~cm}$ ) & 189 & 75.6 & 5 & 2.0 & 194 & 77.6 & \multirow{2}{*}{$<0.001 *$} \\
\hline Difficult intubation $(\mathrm{SMD} \leq 13.5 \mathrm{~cm})$ & 35 & 14.0 & 21 & 8.4 & 56 & 22.4 & \\
\hline Easy intubation $(\mathrm{TMD}>6.5 \mathrm{~cm})$ & 195 & 78.0 & 3 & 1.2 & 198 & 79.2 & $<0.001^{*}$ \\
\hline
\end{tabular}

TABLE 3. Comparison of sensitivity, specificity and positive and negative cut-off values of all groups

\begin{tabular}{lcccc}
\hline & Sensitivity & Specificity & PPV & NPV \\
\hline MMT & 73.08 & 90.62 & 47.50 & 96.67 \\
ULBT & 57.69 & 99.11 & 36.24 & 95.28 \\
IID & 84.62 & 83.04 & 36.70 & 37.89 \\
SMD & 80.77 & 84.37 & 37.50 & 97.42 \\
TMD & 88.46 & 87.05 & 44.23 & 98.48 \\
\hline
\end{tabular}

MMT: Modified Mallampati test; ULBT: Upper-Lip Bite test; IID: interincisor distance; SMD: sternomental distance; TMD: thyromental distance; PPV: positive predictive value; NPV: negative predictive value 
12 cases were identified as probable difficult intubations. The difference between the possible cases of difficult intubation and the actual number of difficult intubations was statistically significant $(\mathrm{p}<0.001$, Table 4$)$. The sensitivity was $46.15 \%$, the specificity was $100.00 \%$, the PPV was $100.00 \%$ and the NPV was $94.12 \%$ (Table 5 ). When ULBT was combined with the MMT, IID and SMD, in the quaternary test, 15 cases of easy intubation were identified as difficult intubations, while 12 cases were identified as probable difficult intubations (Table 4). A statistically significant difference was detected between possible cases of difficult intubation and the actual number of difficult intubations $(\mathrm{p}<0.001$; sensitivity: 43.31\%; specificity: 100.00\%; PPV: 100.00; NPV: 93.72\%;
Table 5). If the five tests were combined, 15 cases of easy intubation were identified as difficult intubations, while 12 cases were identified as probable difficult intubations (Table 4). The difference between the possible cases of difficult intubation and the actual number of difficult intubations was statistically significant ( $<<0.001$; sensitivity: $43.31 \%$; specificity: 100.00\%; PPV: 100.00\%; NPV: 93.72\%; Table $5)$.

There was moderate agreement between the Cormack-Lehane classification and the ULBT, MMT, IID, SMD combination and the ULBT, MMT, IID, SMD, TMD combination, while there was high agreement between the Cormack-Lehane classification and other assessments (Table 4).

TABLE 4. Comparison of the group combinations

\begin{tabular}{|c|c|c|c|c|c|c|c|c|c|}
\hline & & \multicolumn{2}{|c|}{$\begin{array}{c}\text { Easy intubation } \\
\text { (Cormack } \\
\text { Grade I-II) }\end{array}$} & \multicolumn{2}{|c|}{$\begin{array}{c}\text { Difficult intubation } \\
\text { (Cormack } \\
\text { Grade III-IV) }\end{array}$} & \multicolumn{2}{|c|}{ Total } & \multirow[t]{2}{*}{$\mathrm{p}^{*}$} & \multirow[t]{2}{*}{ К } \\
\hline & & $\%$ & $\mathrm{n}$ & $\%$ & $\mathrm{n}$ & $\%$ & $\mathrm{n}$ & & \\
\hline & & 89.60 & 26 & 10.40 & 250 & 100 & 224 & & \\
\hline \multirow{2}{*}{$\mathrm{ULBT}+\mathrm{MMT}$} & Easy intubation & 224 & 89.60 & 11 & 4.40 & 235 & 94.00 & \multirow{2}{*}{0.001} & \multirow{2}{*}{0.710} \\
\hline & Difficult intubation & 0 & 0.00 & 15 & 6.00 & 15 & 6.00 & & \\
\hline \multirow{2}{*}{$\mathrm{ULBT}+\mathrm{IID}$} & Easy intubation & 224 & 89.60 & 14 & 5.60 & 238 & 95.20 & \multirow{2}{*}{$<0.001$} & \multirow{2}{*}{0.606} \\
\hline & Difficult intubation & 0 & 0.00 & 12 & 4.80 & 12 & 4.80 & & \\
\hline \multirow{2}{*}{ ULBT + SMD } & Easy intubation & 223 & 89.20 & 12 & 4.80 & 235 & 94.00 & \multirow{2}{*}{0.003} & \multirow{2}{*}{0.657} \\
\hline & Difficult intubation & 1 & 0.40 & 14 & 5.60 & 15 & 6.00 & & \\
\hline \multirow{2}{*}{ ULBT + TMD } & Easy intubation & 223 & 89.20 & 11 & 4.40 & 234 & 93.60 & \multirow{2}{*}{0.006} & \multirow{2}{*}{0.690} \\
\hline & Difficult intubation & 1 & 0.40 & 15 & 6.00 & 16 & 6.40 & & \\
\hline \multirow{2}{*}{$\mathrm{ULBT}+\mathrm{MMT}+\mathrm{IID}$} & Easy intubation & 224 & 89.60 & 14 & 5.60 & 234 & 95.20 & \multirow{2}{*}{$<0.001$} & \multirow{2}{*}{0.606} \\
\hline & Difficult intubation & 0 & 0.00 & 12 & 4.80 & 12 & 4.80 & & \\
\hline \multirow{2}{*}{$\mathrm{ULBT}+\mathrm{MMT}+\mathrm{IID}+\mathrm{SMD}$} & Easy intubation & 224 & 89.20 & 15 & 6.00 & 234 & 96.60 & \multirow{2}{*}{$<0.001$} & \multirow{2}{*}{0.568} \\
\hline & Difficult intubation & 0 & 0.00 & 11 & 4.40 & 12 & 4.40 & & \\
\hline \multirow{2}{*}{$\mathrm{ULBT}+\mathrm{MMT}+\mathrm{IID}+\mathrm{SMD}+\mathrm{TMD}$} & Easy intubation & 224 & 89.20 & 15 & 6.00 & 234 & 96.60 & \multirow{2}{*}{$<0.001$} & \multirow{2}{*}{0.568} \\
\hline & Difficult intubation & 0 & 0.00 & 11 & 4.40 & 12 & 4.40 & & \\
\hline
\end{tabular}

TABLE 5. Comparison of group combinations according to sensitivity, specificity and positive and negative predictive values

\begin{tabular}{|c|c|c|c|c|c|}
\hline & Sensitivity & Specificity & PPV & NPV & $\mathrm{p}^{*}$ \\
\hline $\mathrm{ULBT}+\mathrm{MMT}$ & 57.69 & 100.00 & 100 & 95.32 & 0.001 \\
\hline $\mathrm{ULBT}+\mathrm{IID}$ & 46.15 & 100.00 & 100 & 94.12 & $<0.001$ \\
\hline $\mathrm{ULBT}+\mathrm{SMD}$ & 57.69 & 99.55 & 93.75 & 95.30 & 0.003 \\
\hline $\mathrm{ULBT}+\mathrm{TMD}$ & 93.75 & 95.30 & 57.69 & 99.55 & 0.006 \\
\hline $\mathrm{ULBT}+\mathrm{MMT}+\mathrm{IID}$ & 46.15 & 100.00 & 100 & 94.12 & $<0.001$ \\
\hline $\mathrm{ULBT}+\mathrm{MMT}+\mathrm{IID}+\mathrm{SMD}$ & 42.31 & 100.00 & 100 & 93.72 & $<0.001$ \\
\hline $\mathrm{ULBT}+\mathrm{MMT}+\mathrm{IID}+\mathrm{SMD}+\mathrm{TMD}$ & 42.31 & 100.00 & 100 & 93.72 & $<0.001$ \\
\hline
\end{tabular}

${ }^{*} \mathrm{p}<0.05$ statistically significant according to McNemar test; ULBT: Upper-Lip Bite test; MMT: Modified Mallampati test; IID: interincisor distance; SMD: sternomental distance; TMD: thyromental distance; PPV: positive predictive value; NPV: negative predictive value 


\section{DISCUSSION}

Unanticipated difficult endotracheal intubation in the anaesthesia practice is an undesirable and life-threatening condition. The most common cause of anaesthesia-related morbidity and mortality is airway inability after induction (15). Shiga et al. (5) identified difficult intubation cases at a frequency of $5.8 \%$ in a meta-analysis comprising 50.760 patients. Iohom et al. (6) identified difficult intubation in $9 \%$ of 212 patients. Mallampati et al. (16) predicted difficult intubation in 28 out of 210 cases in direct laryngoscopy, a rate of $13.3 \%$. In our study, the incidence of difficult intubation was $10.4 \%$. The main reason for this situation may be the increase in the incidence of a physiologically difficult airway due to airway oedema in pregnant women. Another reason is that the preanaesthetic evaluation of all the cases was not performed by the same person.

Whether laryngoscopy will be easy or difficult may be predicted by using the Mallampati classification (16). Although the Mallampati test is not considered as reliable as in the past, due to interobserver variability, the patient's position, mounding of the tongue during the procedure or neck mobility, it is still a convenient and practical method that is easily applied at the bedside. Merah et al. (4) performed five bedside monitoring tests in 80 obstetric patients and found $87.1 \%$ sensitivity and $99.6 \%$ specificity of the Mallampati test. They concluded that the Mallampati test could be used to predict difficult intubation. Frerk (17) stated that MMT was not specific for routine use, because it had high sensitivity but produced many false-positive $(1 / 5)$ results with $81.2 \%$ sensitivity and $81.5 \%$ selectivity. In our study, we found the MMT to have $73.08 \%$ sensitivity, 90.62\% specificity, $47.50 \%$ PPV and $96.67 \%$ NPV. Possible explanations for the disparate results are the number of cases, failure to establish good communication with patients, different patient groups and interobserver variability.

The ULBT is a new, simple and easily applied test. Eberhart et al. (18) found $28.2 \%$ sensitivity, $92.5 \%$ specificity, $33.6 \%$ PPV and 90.6\% NPV in their study. Hester et al. (13) found $55 \%$ sensitivity, $97 \%$ specificity and $83 \%$ PPV and stated that the test was applicable because it was easy to apply. For the ULBT, Salimi et al. (19) found 70\% sensitivity, $93 \%$ specificity, $39 \%$ PPV and 98\% NPV in their study. We found $57.69 \%$ sensitivity, $99.11 \%$ specificity, $88.24 \%$ PPV and $95.28 \%$ NPV for the ULBT. In a recent study conducted by Khan et al. (20) they found $78.91 \%$ sensitivity, $91.96 \%$ specificity, $91.05 \%$ PPV and $98.8 \%$ NPV for ULBT. The investigators stated that the ULBT alone was not sufficient to predict difficult intubation with these results but would be useful for distinguishing easy intubation cases because it had high sensitivity and negative cut-off values. The practicality of the ULBT and the correlation of its results with those of the MMT demonstrate its usefulness in predicting difficult intubation in pregnant women. Moreover, Allahyary et al. (11) found that the ULBT as a single test was highly sensitive (94.6\%) and specific (97.6\%), and as a result, the ULBT was reported to be a valuable marker for predicting difficult laryngoscopy.

Yildiz et al. (10) found a significant difference between the IID values of the difficult and easy laryngoscopy cases when they confirmed the cut-off value as $45 \mathrm{~mm}$ for IID. Wilson et al. (21) found an increased risk of difficult laryngoscopy in patients with IID smaller than $5 \mathrm{~cm}$. Assk et al. (22) found that patients who had IID values less than $30 \mathrm{~mm}$ had statistically significantly higher cut-off values than those with higher IID values. This study reported a sensitivity of $36 \%$, a specificity of $81 \%$, PPV of $35 \%$ and NPV of $81 \%$. For the IID measurement for predicting difficult intubation in pregnant women, we found $84.62 \%$ sensitivity, $83.04 \%$ specificity, $36.67 \%$ PPV and $97.89 \%$ NPV. We propose that lower threshold values and the different numbers of cases in other studies explain the disparate results between the studies.

The extension of the head is an important parameter in predicting whether the intubation will be easy or difficult. SMD may be an indicator of head and neck mobility (23). Shiga et al. (5) found a moderate level of sensitivity for SMD (62\%), 82\% specificity, higher PPV and lower NPV compared with the other tests and argued that it was the best test for predicting difficult intubation. Al Ramadhani et al. (23) evaluated the Cormack grade classification and SMD of 523 patients who underwent emergency or elective caesarean section and considered an SMD of $13.5 \mathrm{~cm}$ or less as the threshold for predicting difficult intubation. The authors also reported a sensitivity of $66.7 \%$, a specificity of $71.1 \%$, PPV of $7.6 \%$ and NPV of $98.4 \%$. Savva (24) reported that SMD should be used as the single objective indicator of difficult intubation. This study reported a sensitivity of $82.4 \%$, a specificity of $88.6 \%$ and a PPV of $26.9 \%$, provided that SMD was less than $12.5 \mathrm{~cm}$. In our study, the cut-off value for SMD was $13.5 \mathrm{~cm}$, the sensitivity was $80.77 \%$, the specificity was $84.37 \%$, PPV was $37.50 \%$ and NPV was $97.42 \%$. Therefore, we believe that the disparate results may have been due to the use of different cut-off values and the failure to bring the patient's head into sufficient extension.

Yildiz et al. (10) reported that the probability of a difficult intubation is high when TMD is between 6 and $6.5 \mathrm{~cm}$ and impossible when it is below $6 \mathrm{~cm}$. Frerk (17) mentions the probability of a difficult intubation when TMD is under $7 \mathrm{~cm}$. We identified the cut-off value for TMD as $6.5 \mathrm{~cm}$. Tse et al. (25) reported that TMD $\leq 7 \mathrm{~cm}$ was not a good predictor of difficult intubation preoperatively, as it had a low sensitivity (32\%), PPV of $20 \%$, high specificity ( $80 \%)$ and NPV of $89 \%$. 
Wong and Hung (26) conducted a study to assess the prevalence and prediction of difficult intubation in Chinese pregnant and non-pregnant patients. They found no difference in the rates of difficult intubation and concluded combination of predictive tests for assessing difficult intubation. They used the IID and TMD in their study and found a statistically significant difference in IID values. The authors also found sensitivity and specificity as high as $71 \%$ and $92 \%$, respectively, when TMD $<5.5 \mathrm{~cm}$ was taken, whereas PPV was only found to be $11 \%$. We found that the sensitivity for TMD was $88.46 \%$, the specificity was $87.05 \%$, PPV was $44.23 \%$ and NPV was $98.48 \%$.

Since a single test is not sufficient to predict difficult intubation, it was reported that the most accurate results would be obtained by a combination of tests (5). In the study by Iohom et al. (6), when used alone, the MMT, TMD and SMD tests were weak in terms of sensitivity, specificity and PPV. When MMT was combined with TMD or SMD, the sensitivity decreased, but the negative cut-off value remained at $93 \%$. They found that the specificity and positive cut-off value for the MMT with the TMD increased from $89 \%$ to $100 \%$ and increased from $27 \%$ to $100 \%$ with SMD. They reported that, when TMD and SMD were used together, the sensitivity decreased (33\%), the specificity increased (98\%) and PPV increased (67\%). They concluded that the MMT, TMD and SMD tests would be useful routine preoperative tests in predicting difficult intubation. Ylldiz et al. (10) found that the combination of MMT and TMD had a high positive cut-off value (50\%), as the combination of MMT, IID and TMD had the highest positive cut-off value among all combinations (66.7\%). Frerk (17) concluded that the combination having the highest sensitivity and selectivity was the MMT and TMD measurement. in conjunction with the MMT, the sensitivity was $57.69 \%$, the specificity was $100.00 \%$, PPV was $100.00 \%$ and NPV was $95.32 \%$. When TMD and the ULBT were used together, the sensitivity was $93.75 \%$, specificity was $95.30 \%$, PPV was $57.69 \%$ and NPV was $99.55 \%$.

When all combinations are evaluated in the decision of difficult intubation, the combination of the ULBT and TMD methods is more suitable to use than other methods alone to predict difficult intubation in pregnant women.

Financial Disclosure: No financial disclosure was declared by the authors.

Conflict of Interest: No conflict of interest was declared by the authors.

\section{REFERENCES}

1. Hawkins JL, Chang J, Palmer SK, Gibbs CP, Callaghan WM. Anesthesiarelated maternal mortality in the United States: 1979-2002. Obstet Gynecol 2011;117:69-74.

2. Hawthorne L, Wilson R, Lyons G, Dresner MFailed intubation revisited: 17-yr experience in a teaching maternity unit. Br J Anaesth 1996;76:680-4.

3. Caplan RA, Posner KL, Ward RJ, Cheney FW. Adverse respiratory events in anesthesia: a closed claims analysis. Anesthesiology 1990;72:828-33.

4. Merah NA, Foulkes-Crabbe DJ, Kushimo OT, Ajayi PA. Prediction of difficult laryngoscopy in a population of Nigerian obstetric patients. West Afr J Med 2004;23:38-41

5. Shiga T Wajima Z, Inoue T, Sakamoto A. Predicting difficult intubation in apparently normal patients: a meta-analysis of bedside screening test performance. Anesthesiology 2005;103:429-37.

6. Iohom G, Ronayne M, Cunningham AJ. Prediction of difficult tracheal intubation. Eur J Anaesthesiol 2003;20:31-6.

7. Samsoon GL, Young JR. Difficult tracheal intubation: a retrospective study. Anaesthesia 1987;42:487-90.

8. Karkouti K, Rose DK, Ferris LE, Wigglesworth DF, Meisami-Fard T, Lee H. Inter-observer reliability of ten tests used for predicting difficult tracheal intubation. Can J Anaesth 1996;43:554-9.

9. Adamus M, Jor O, Vavreckova T, Hrabalek L, Zapletalova J, Gabrhelik T, et al. Inter-observer reproducibility of 15 tests used for predicting difficult intubation. Biomed Pap Med Fac Univ Palacky Olomouc Czech Repub 2011;155:275-81.

10. Yildiz TS, Culha TH, San S, Solak M, Toker M. Which Tests are the Most Reliable for Predicting Difficult Intubation? Turkish Journal of Anesthesiology and Reanimation. 2006;34:162-8.

11. Allahyary E, Ghaemei SR, Azemati S. Comparison of six methods for predicting difficult intubation in obstetric patients. IRCMJ 2008;10:197204.

12. Khan ZH, Kashfi A, Ebrahimkhani E. A comparison of the upper lip bite test (a simple new technique) with modified Mallampati classification in predicting difficulty in endotracheal intubation: a prospective blinded study. Anesth Analg 2003;96:595-9.

13. Hester CE, Dietrich SA, White SW, Secrest JA, Lindgren KR, Smith T. A comparison of preoperative airway assessment techniques: the modified Mallampati and the upper lip bite test. AANA J 2007;75:177-82.

14. Cormack RS, Lehane J. Difficult tracheal intubation in obstetrics. Anaesthesia 1984;39:1105-11

15. American Society of Anesthesiologists Task Force on Management of the Difficult Airway. Practice guidelines for management of the difficult airway: an updated report by the American Society of Anesthesiologists Task Force on Management of the Difficult Airway. Anesthesiology 2003;98:1269-77.

16. Mallampati SR, Gatt SP, Gugino LD, Desai SP, Waraksa B, Freiberger D, et al. A clinical sign to predict difficult tracheal intubation: a prospective study. Can Anaesth Soc J 1985;32:429-34.

17. Frerk CM. Predicting difficult intubation. Anaesthesia 1991;46:1005-8.

18. Eberhart LH, Arndt C, Cierpka T, Schwanekamp J, Wulf H, Putzke C. The reliability and validity of the upper lip bite test compared with the Mallampati classification to predict difficult laryngoscopy: an external prospective evaluation. Anesth Analg 2005;101:284-9.

19. Salimi A, Farzanegan B, Rastegarpour A, Kolahi AA. Comparison of the upper lip bite test with measurement of thyromental distance for prediction of difficult intubations. Acta Anaesthesiol Taiwan 2008;46:61-5.

20. Khan ZH, Mohammadi M, Rasouli MR, Farrokhnia F, Khan RH. The diagnostic value of the upper lip bite test combined with sternomental distance, thyromental distance, and interincisor distance for prediction of easy laryngoscopy and intubation: a prospective study. Anesth Analg 2009;109:822-4. 
21. Wilson ME, Spiegelhalter D, Robertson JA, Lesser P. Predicting difficult intubation. Br J Anaesth 1988; 61:211-6.

22. Asık I, Goktug A, Canakc1 N. The relationship of different evaluation tests with difficult intubation. Journal of Anesthesiology 2000;8:188-92.

23. Al Ramadhani S, Mohamed LA, Rocke DA, Gouws E. Sternomental distance as the sole predictor of difficult laryngoscopy in obstetric anaesthesia. Br J Anaesth 1996;77:312-6.
24. Savva D. Prediction of difficult tracheal intubation. Br J Anaesth 1994;73:149-53.

25. Tse JC, Rimm EB, Hussain A. Predicting difficult endotracheal intubation in surgical patients scheduled for general anesthesia: a prospective blind study. Anesth Analg 1995;8:254-8.

26. Wong SH, Hung CT. Prevalence and prediction of difficult intubation in Chinese women. Anaesth Intensive Care 1999;27:49-52. 